\title{
Multidisciplinary review of logistics and supply chain research- Application of scientific measurement analysis in related fields
}

\author{
Chung-Lien Pan*1, JiaLong Li $^{1}$, XiaoLing Huang ${ }^{1}$, XuanFan Guo ${ }^{1}$, LiHong Zhang ${ }^{1}$ \\ ${ }^{1}$ Nanfang College of Sun Yat-sen University, School of Accounting, Guangzhou, Guangdong
}

\begin{abstract}
The process of globalization and digitization has continuously increased customer requirements for logistics costs and services. Issues related to the development of logistics and supply chain has received great attention. However, considering the factors of timeliness and systemic, previous studies could no longer meet the latest needs. To fill these gaps, this article reviews the 833 articles collected from the Web of Science (WoS) database from 1989 to March 2020 and mainly analyzes annual trends, top organizations, top published countries, author keywords, and main sources and disciplines, using the concepts of system thinking and knowledge-intermediary to summarize the research results. The results show that the research on logistics and supply chain is in a period of rapid development, and presents a decentralized and diversified development trend. These data and images provide a comprehensive picture of the development of the digital business economy, aiming to help researchers, funding agencies, policymakers, and industry professionals grasp the timely situation about the discipline, thus establishing a systematic understanding.
\end{abstract}

\section{Introduction}

With the development of the Internet and the Internet of Things (IoT), the importance of logistics and supply chain has become increasingly prominent and used as a means to improve competitiveness or maintain competitiveness in this challenging global environment [1]. Craighead, C. W. believes that the focus of market competition has evolved from individual company competition to competition between the entire supply chain, makes effective supply chain reengineering particularly important [2].

The previous literature in related fields lacks a systematic review. Traditional data and analysis articles usually focus on resource planning and algorithm optimization, as well as their impact and changes on logistics and supply chain applications. For example Chae, B. (Kevin). proposed a novel analysis framework (Twitter Analytics) to analyze supply chain tweets, and deeply explored the potential role of Twitter in supply chain practice and research [3]. Gunasekaran, A et al. developed and tested a Big Data and Predictive Analysis (BDPA) model to explain the impact of BDPA assimilation on the supply chain (SCP) and organizational performance (OP) [4]. At the same time, the advent of the digital age has greatly expanded scholars' research horizons. Lee, I., \& Lee, K. believe that the application of the Internet of Things (IoT) is helpful for information sharing and collaboration. In the field of the supply chain, information sharing and collaboration can enhance situational awareness and avoid information delay and distortion [5]. Ellis, D. I et al. discuss a series of spectroscopy and spectroscopy-based methods and establishes a systemlevel solution to the areas of the food supply chain that are susceptible to food crime [6]. Kshetri, N. believes that the arrival of blockchain will change supply chain activities, and using blockchain in supply chain activities can increase transparency and accountability [7]. The continuous emergence of research in these emerging fields has changed the overall look of traditional research. So, it's necessary to conduct a review with the latest data.

Logistics and supply chain management is an emerging discipline that intersects multiple disciplines such as transportation, military science, computer science, marketing, sociology, economics, and management science [8]. Only by collecting comprehensive information across multidiscipline can we draw a full picture of this industry. This article collected the data from the WoS database in March 2020. Hoping to provide a comprehensive image that intuitively explains the relationship between logistics supply chain and related research areas, identifies the latest developments and discussions on this topic.

\section{Data and methods}

This article aims to provide a literature review on the subject of "logistics, supply chain". In order to obtain relevant documents, we first collect documents from WoS and make the following queries:

$\mathrm{TS}=$ ("Block chain" OR "Internet of things" OR "IoT" OR "Big data" OR "Information system and Technology") AND TS = ("Supply chain" OR "Logistics") AND SU = ("Business \& Economics" OR "Government \& Law" OR "Social Sciences" OR "Management" OR

*Corresponding author. Email: peter5612@gmail.com 
"Communication" OR "Technology")

By March 2020, a total of 833 articles were collected (including SCI-EXPANDED SSCI CPCI-S CPCI-SSH and ESCI). And also conducted bibliographic coupling (based on clustering) and co-citation analysis research for tracking frontier fields, using Python and VOSviewer software for clustering and visualization.

\section{PRESEARCH FINDINGS}

The above mapping results show some findings, including annual trends, publications, institutions, countries, and themes.

\subsection{Annual trends}

As can be seen from Figure 1, the number of publications has shown an upward trend since 2008, reaching a peak of 197 in 2018, and maintained this number the following year. Figure 2 shows that the number of related citations with a stronger growth rate. Among them, 2016 is an important turning point. From this, the number of citations in the literature began to double year by year. Such a violent momentum is relatively rare, which reflects the increasing importance of the subject and the academic interest that researchers have soared.

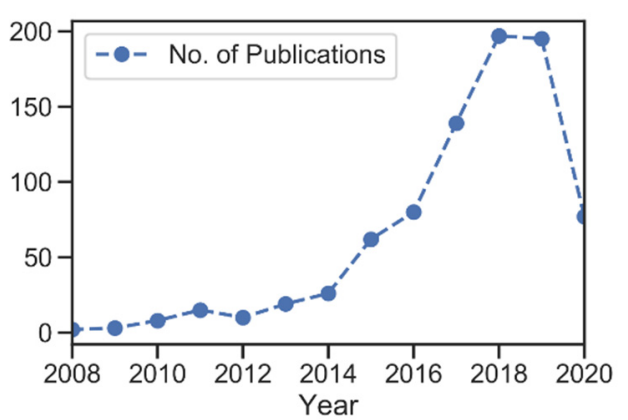

Fig1. The number of publications

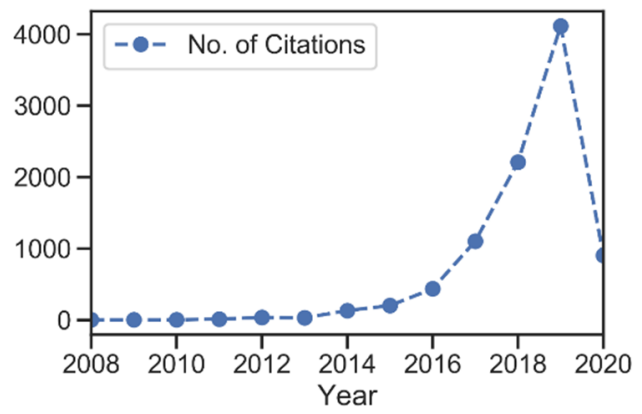

Fig2. The number of citations

\subsection{Top organizations}

To further explore the research status of logistics and supply chain, and find out the key subject leaders, industry formulators, and similar schools of research, this paper analyzes the top institutions, including organizations, companies, think tanks, universities, and so on.

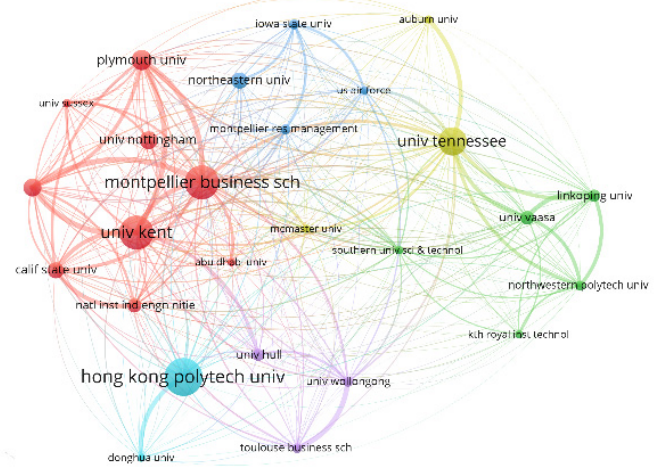

Fig3. Top organizations

As shown in Figure 3, almost all of the top 30 institutions are occupied by universities, with the only exception being the US Air Force. From the image distribution, we can see that the red interval dominated by Montpellier Business School, The University of Kent, and The University of Plymouth has a strong correlation strength, which indicates that these institutions are in the state of academic frontier or academic cohesion. In addition, The University of Tennessee, The Hong Kong Polytechnic University, Northeastern University are also very important, but their research is more independent and has no widely connected cluster.

\subsection{Top published countries}

Geographical distribution is another way to distinguish the relationship between clusters, mainly showing the central intensity of field research. It represents the extent to which different scientific groups that are part of the center are brought together, reflecting the concept "collision" between scientific centers and scientific members across disciplines or geographic boundaries [9].

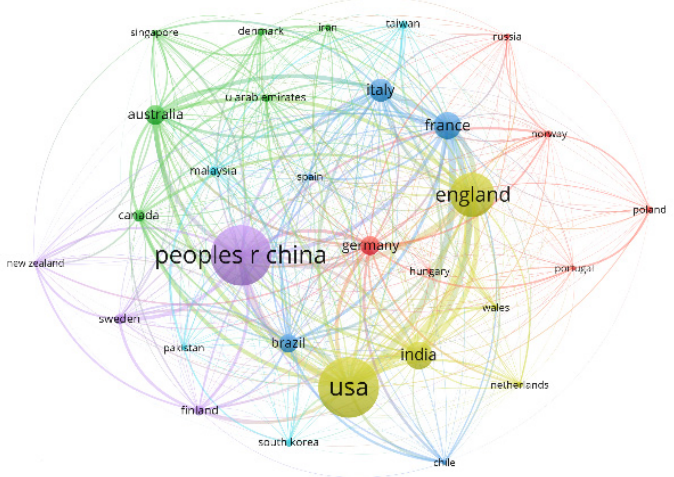

Fig4. Top countries

Among the clusters in Figure 4, dominate countries from high to low, including the United States, China, France, and Germany. Compared with the distribution trend in Figure 3, the clusters in Figure 4 show a more distributed distribution trend, that is to say, such results confirm that the research perspectives of logistics and supply chain are diverse and there are no particularly stable schools or research centers. 


\subsection{Author keywords}

In order to explore the correlation and strength between items, according to the co-occurrence based on bibliographic keywords (author keywords). Figure 5 shows the clustering results of the top 40 items. These visual keywords have been pre-processed by the thesaurus compiled by the author, combining similar concepts into a unified terminology, such as IoT and Internet of things.

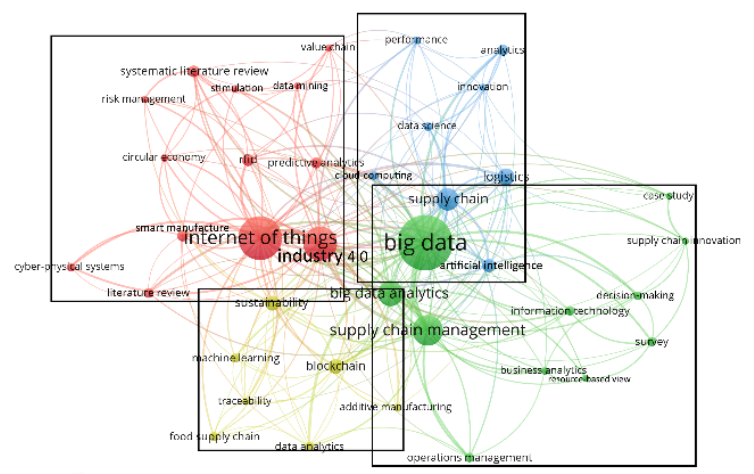

Fig5. A map based on bibliographic keyword co-occurrence (author keywords)

Academia generally believes that logistics and supply chain are similar concepts and integrated with development [10], and the image display results also prove this. The two concepts are nearby and are connected to similar projects. In terms of the strength of contact with keywords, logistics, and supply chain correspond to "big data, internet of things, industry 4.0, blockchain" and "big data, internet of things, AI, cloud computing." The cluster analysis of the keyword graph is helpful to identify four main clusters, as follows:

- $\quad$ Red "internet of things" cluster:

The red cluster is dominated by "internet of things" and "industry $4.0 "$ ". It can be seen from the figure that relevant research fields have been mature. A systematic literature review is carried out, which involves the fields of rulemaking, market research, sociological research, business. In addition, the emergence of multiple research perspectives, including "radio frequency identification (RFID) ", "predictive analysis", "cyber-physical systems", "smart manufacture", "risk management", etc., proves that the research in this field is not yet saturated and remains a hot spot in this field.

- Green "big data" cluster

In the green cluster, it shows the value of digitization to research. "big data", "big data analysis" and "supply chain management" is the most active research. Other topics include "operations management", "information technology", "decision making", and "resource-based view". Compared with other clusters, green clusters have the most related items. It is not difficult to see that under the influence of digital trends, there are more extensive research opportunities in data-related research.

- Blue "supply chain" cluster

As shown in the blue cluster. "Logistics", "supply chain" and "cloud computing", "artificial intelligence", and "data science" have greater link strength, and together form a mature interdisciplinary research system. At the same time, the emergence of the keywords such as "innovation" and "performance" shows that the relevant research in the blue cluster pays attention to practical practice and practical benefits, which has a very obvious pragmatism color.

- Yellow "sustainability" clusters

The yellow cluster shows some pioneering research, including "blockchain", "machine learning", "traceability", and "addicted manufacturing". Although these themes appeared late, it is undeniable that they represent the forefront of field research and have the potential to trigger disruptive revolutions. Feng Tian. believes that in future research, with the rapid development of blockchain, it is the development trend of the logistics industry to establish a decentralized system with completely reliable information [11].

\subsection{Main sources and disciplines}

As shown in Figure 6, among the published journals, "International Journal of Production Research", "Journal of Cleaner Production", "Annals of Operations Research" and "Sustainability" are interconnected and occupy key positions. More specifically, the first-ranked journals are management disciplines, and the third and fourth disciplines are engineering disciplines. At the same time, these two disciplines are also the most appeared. In a smaller extend, there are also environmental science and ecology journals, including the aforementioned "Journal of Cleaner Production" and "British Food Journal".

Among the top 30 journals, journals specializing in logistics and supply chain management account for only a small part, including "Journal of Business Logistics", "International Journal of Logistics Management", "Transportation Research Part E-Logistics And Transportation Review", "International Journal of Physical Distribution \& Logistics Management" and " Supply Chain Management-An International Journal". These journals have a long history and maintain considerable influence in related fields. It is worth noting that interdisciplinary research journals such as "Computer \& Operations Research", "Expert Systems with Applications", "Discrete Dynamics in Nature and Society" and "Symmetry-Basel" also account for a considerable proportion. In contrast, these journals are younger. Their emergence is reflected in the trend of the development of traditional logistics and supply chain disciplines to the outside world.

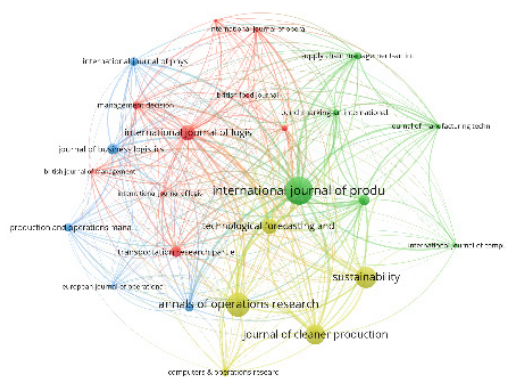

Fig6. Top publication sources: a bibliographic coupling relationship network visualization 


\section{CONCLUSION}

In academia, the heat of logistics and supply chain is constantly rising. The correct and effective direction is the premise of improving research efficiency. This article systematically organizes the research status of digital technology in the logistics and supply chain industries through big data analysis and visualization procedures.

The results of the study show that: (1) The number of research documents on logistics and supply chain is increasing rapidly. (2) The overall development of the discipline shows a trend from traditional fields to diversified research. (3) The majority of institutions studying this subject are universities, and the overall distribution is relatively scattered. (4) In the related fields, there are not only mature topics such as big data, the Internet of Things, and Industry 4.0, but also emerging fields such as sustainability, blockchain, and additional manufacturing research, which have both heat and potential.

As the results of the scientific measurement analysis carried out in this article, with the continuous integration of digital technology and logistics and supply chain and the emergence of new research such as sustainability, environmental science, the depth and breadth of related fields have been expanded to a brand new grade. The latest data and images provided in this paper will help scholars to observe the development trend of the industry intuitively, track the frontier research in the field, and establish systematic thinking.

\section{Acknowledgments}

We thank our colleague Han-Teng Liao and Zhichao Xu who provided expertise and insight into the bibliometric data that greatly helped the research.

\section{REFERENCES}

1. Mentzer, J. T., DeWitt, W., Keebler, J. S., Min, S., Nix, N. W., Smith, C. D., \& Zacharia, Z. G. (2001). Defining supply chain management. Journal of Business logistics, 22(2), 1-25.

2. Craighead, C. W., Hult, G. T. M., \& Ketchen, D. J. (2009). The effects of innovation-cost strategy, knowledge, and action in the supply chain on firm performance. Journal of Operations Management, 27(5), 405-421. doi:10.1016/j.jom.2009.01.002

3. Chae, B. (Kevin). (2015). Insights from hashtag \#supplychain and Twitter Analytics: Considering Twitter and Twitter data for supply chain practice and research. International Journal of Production Economics, $165, \quad 247-$ 259. doi:10.1016/j.ijpe.2014.12.037

4. Gunasekaran, A., Papadopoulos, T., Dubey, R., Wamba, S. F., Childe, S. J., Hazen, B., \& Akter, S. (2017). Big data and predictive analytics for supply chain and organizational performance. Journal of Business Research, 70, 308-317. doi:10.1016/j.jbusres.2016.08.004
5. Lee, I., \& Lee, K. (2015). The Internet of Things (IoT): Applications, investments, and challenges for enterprises. Business Horizons, 58(4), 431440. doi:10.1016/j.bushor.2015.03.008

6. Ellis, D. I., Muhamadali, H., Haughey, S. A., Elliott, C. T., \& Goodacre, R. (2015). Point-and-shoot: rapid quantitative detection methods for on-site food fraud analysis - moving out of the laboratory and into the food supply chain. Analytical Methods, 7(22), 94019414. doi:10.1039/c5ay02048d

7. Kshetri, N. (2018). 1 Blockchain's roles in meeting key supply chain management objectives. International Journal of Information Management, 39, 80-89. doi:10.1016/j.ijinfomgt.2017.12.005

8. AiHu Wang. (2009). review of the development of logistics and supply chain management home and abroad. Journal of South China University of Technology(Social Science Edition), 011(002), 36-42.

9. Bozeman, B. and Corley, E. (2004) 'Scientists' Collaboration Strategies: Implications for Scientific and Technical Human Capital', Research Policy, 33: 599-616.

10. Zhen Jin. (2000). Integration of logistics and supply chain management. Logistics Technology, 000(003), 33.

11. Feng Tian. (2016). An agri-food supply chain traceability system for China based on RFID \& blockchain technology. 2016 13th International Conference on Service Systems and Service Management

(ICSSSM). doi:10.1109/icsssm.2016.7538424 